\title{
Diagnosis of Infectious or Inflammatory Psychosyndromes
}

\author{
Karl Bechter
}

Clinic for Psychiatry and Psychotherapy II, Ulm University, Germany

\begin{abstract}
Before an outline of the process of diagnosis and differential diagnosis in infectious and/or inflammatory psychosyndromes is given, a more general overview onto the approach to organic psychosyndromes seems useful, because in both entities similar principles of causality conclusion are applied. Correlation does not demonstrate causality. Therefore the principles and consensus recommendations, and limitations of causal inference to categorize psychosyndromes as being 'organic', is to be discussed in detail.
\end{abstract}

Keywords: Psychosyndromes, psychoses, syndromes.

\section{CATEGORIES OF ORGANIC PSYCHOSYN- DROMES}

The chapter F0 of ICD-10 involves all organic psychosyndromes with the exception of alcohol- and substancerelated organic disorders. This catalogue outlines various types of dementias and deliria and many other types of organic-based psychosyndromes, ranging from hallucinosis or catatonia, or delusion, or affective, or manic, or bipolar depressed, or mixed affective, or anxiety, or dissociative, or emotional, to minor cognitive disturbances. An encephalitic syndrome is not described, but a post-encephalitic syndrome. Few criteria to diagnose an organic psycho-syndrome are formulated including mainly two points: 1 . the demonstration of a cerebral disease, or trauma, or dysfunction, or 2 . the prevalence of a systemic disease affecting the body known to possibly associate with one of the organic psychosyndromes, plus a relevant time-relationship (weeks or few months) in between this organic factor and onset of psychosyndrome. Further supporting criteria might be improvement or dissolving of psychosyndromes with improvement of the assumed underlying organic cause, and/or a lack of another causality. Clear operational criteria for causal inference are apparently not available. So in reality the diagnosis of an organic psychosyndrome is basically left to the individual arbitrary opinion of the diagnosing clinician. Such is surely well working in acute and subacute organic diseases with a rather clear beginning and/or clear dissolving of symptoms. However, in slow onset insults to the brain any causality conclusion remains extremely difficult. In some chronic psychosyndromes the improvement after therapeutic intervention might mark the point for causality inference, as we recently demonstrated in cases with arachnoid cysts associated with organic psychosyndromes in the form of minor personality disorder, which were reversible resp. improved after neurosurgery [1].

*Address correspondence to this author at the Ulm University, Clinic for Psychiatry and Psychotherapy II Ludwig-Heilmeyer-Str. 2, D-89312 Günzburg, Germany; Tel: +49-8221 /96-2540 or 96-00;

Fax: 49-8221 /96-2736; E-mail: Karl.Bechter@bkh-guenzburg.de
A similar description of organic psychosyndromes like the ICD-10 is found in DSM-VI, a major difference however is, that the term 'organic' is eliminated. The term 'delirium' is much more broadly understood and a major criterion to assume a causal relationship between a 'medical disease factor' and the various psychosyndromes, is the presence of a relevant or seemingly relevant time relationship between both.

Relatively well defined criteria are available for the diagnosis of delirium in both systems, i.e. DSM-IV 293.0, ICD10 F05. However, both international classification systems lack clear operational criteria for most of what is traditionally termed organic psychosyndromes with less severe or moderate symptoms. In this broad spectrum of psychosyndromes mainly the experienced clinician's opinion based on the evaluation of findings observations and examinations, is the clue for causality inference.

The most advanced categorization of organic psychosyndromes may be found in G. Huber's textbook [2], which is therefore preferred here. Huber differentiates acute or reversible types versus chronic or irreversible types of organic psychosyndromes. A diagnosis of acute organic psychosyndromes is rather clear when accompanying neurological symptoms were assessed. According to various diagnostic descriptions, be it textbooks, ICD-10, or DSM-IV, the main symptom interpreted as 'organic' represents the disturbances of consciousness. Difficult is the diagnosis of organic psychosyndromes if consciousness is not disturbed, which on the other hand definitely is not an obligate symptom even of the acute exogenic reaction type, first described by Bonhoeffer (see below), and usually not found in chronic organic psychosyndromes. Organic psychosyndromes lacking neurological symptoms and lacking disturbances of consciousness were described as functional psychoses or 'Durchgangssyndrome' (transit syndromes). Durchgangssyndromes include various types of psychopathology, e.g. affective and schizophrenic type psychoses, and a variety of 'minor' psychosyndromes presenting with a broad range of psychiatric symptomatology (see above and [3]). Causality inference in such 
cases is a priori difficult because of the general unspecificity of psychosyndromes to be discussed below.

The proposal of G. Huber to rank organic psychosyndromes according to their validity of causal inference is exemplary, though not yet accepted in the international diagnostic systems: First-rank organic psychosyndromes include deliria, dementia and amnestic syndromes, all of them including disturbed consciousness or severe disturbances of cognitive functioning, such symptoms allowing a rather safe causality inference of organic brain dysfunction yet on a psychopathological level. Second-rank organic psychosyndromes involve various types of organic personality disorders, catatonic or delusional syndrome, hallucinosis, affective syndromes, anxiety, obsessive-compulsive syndromes, but also 'neurasthenia' (this term may in the English literature embrace such terms like fatigue and incapacity syndrome), or solely minor cognitive disturbances. Chronic organic psychosyndromes were by G. Huber according to Kurt Schneider staged into three severity levels, i.e. pseudoneurasthenia (mild), organic personality disorder (medium), and dementia (severe). This staging is also not internationally accepted but in my view a very useful clinical categorization, because giving an evaluated information about the strength of causality or relevance of the respective organic factor for the observed respective psychopathology. This classification holds as a special subgroup also for infectious and inflammatory (autoimmune, toxic, post-traumatic, accompanying) psychosyndromes. Note: The terminologies are often confusing when comparing various sources including certain terms of psychopathology which cannot be outlined here in detail. For example functional psychoses according to G. Huber is a clearly organic syndrome whereas functional syndromes according to Fish's clinical psychopathology [4], means psychogenic syndromes in the old triadic system.

\section{THE UNSPECIFICITY OF ORGANIC PSYCHO- SYNDROMES}

But why is the clinical assessment and causal inference of organic psychosyndromes that difficult? The major reason seems the general unspecificity of psychopathological syndromes associated with organic brain dysfunction, the type of psychopathology being widely irrespective of the underlying specific causality factor. All known etiologies of psychiatric disorders including genetic ones are generally nonspecific [5], i.e. any known specific single etiology can induce a rather similar range of psychiatric syndromes or symptoms, so that on a cross sectional time point of disease the respective syndrome or symptom cannot be specified from the symptom level, though in the severe stages an organic syndrome can be clearly assessed. In lower degree organic psychosyndromes even this primary categorization of 'organic' is tentative. Nevertheless, with increasing severity of an organic process over time more and more characteristic symptoms may evolve in the diseased, eventually leading to some 'relative' specificity as termed in G. Huber's textbook and finally to 'organic' level of symptoms, the latter even on an exclusive psychopathological level then clearly to be categorized as 'organic'.

The traded dichotomy between organic and functional disorders and a third category of psychogenic disorders (=triadic system) appeared outdated [6]. A problem however remained in the present international diagnostic classification, that is the lack to try to differentiate between etiology and pathogenesis, and to neglecting psychogenesis as a third possibility or as interacting factors [7], and widely neglecting the general interdependence between psychological and organic factors in psychopathology [8]. So in conclusion, the problem of a clear categorization of organic psychosyndromes seems not solved yet. For years it was proposed to improve etiological research by including specific biological markers into the diagnostic categorization itself $[5,7,9]$. These approaches remained preliminary. For example in the psychoimmunology field despite a wealth of interesting findings clearly supporting a role of low level neuroinflammation in the causation of some severe psychiatric disorders [10], there is a lack of accepted parameters and criteria to define the role of neuroinflammation in psychiatric disorders. But this is the case in general for organic psychosyndromes at least for the majority of the less severe, i.e. second-rank organic psychosyndromes including pseudoneurasthenia and organic personality disorder. So, in these cases representing a broad range and a relevant quantity of organic psychosyndromes, causality inference is to a large part left to the experienced clinician by traded rules [11]. Apparently the diagnostic crisis in psychiatry and the limited construct validity of DSM-IV and ICD-10 [12] may only step by step be overcome in forthcoming versions of the international diagnostic classification systems.

The acute exogenous reaction type (AERT) described by Bonhoeffer [13] represents a similar pattern of symptoms related to various organic insults to the brain typically associated with disturbances of consciousness. The recently described mixed type encephalopathies (MTE) are very similar to the AERT [14]. The MTE represents a definite organic brain syndrome (delirium), primary diagnosis based on clinical observations, the psychopathology including disturbed consciousness (in most of the time present), and on medical findings. Nevertheless, even with modern technologies it remains very difficult to objectify the diagnosis, which may remain not rarely based exclusively on the observed psychopathological symptoms and causality inferred by time course to some medical problem or factor found in parallel [14]. The underlying causes of MTE are various. This raises the question why psychopathological syndromes are that similar and why organic psychosyndromes are a generally unspecific consequence of organic brain dysfunction. The complexity of the brain itself with extended redundancies of so many functional systems and distributed networks, known from a wealth of neuroscience findings, can apparently explain. This complexity is immanent to brain construction, i.e. distributed networks and redundancy. Redundancy is surely useful from an evolutionary point of view, e.g. to avoid complete failure of systems, in the case of dysfunction. But similar dysfunctions may result from insults at various locations [15], and from differing pathologies often leading to similar symptoms. Nevertheless, to some hotspots more specific symptoms, e.g. well known for motor symptoms, may arise. However, redundancy and wide distribution of functions is especially true for psychic functional networks spanning often throughout the whole brain. Function in such networks is distributed therefore widely unspecific psychopathological 
syndromes arise. With complex investigations into the single case, some differentiation of dysfunction of networks on a biological basis can be achieved [16]. Beyond, brain structure and function varies with neurodevelopment, apparently also changing and determining the type of symptoms observed. Especially early insults into the brain may have enduring consequences depending more from the ages where an insult occurs than from the specificity of the insult [17], such may presumably leading to a 'relative' specific type of symptoms. In insightful studies the differentiation of various organic causal factors for minor organic psychosyndromes in personality disorders has very preliminary been assessed [18].

When considering only one certain neurotransmitter system, for example the serotonergic system in the adult brain, relative unspecificity is also seen on such a system level: it was shown that depressive-like behaviour in animals is counter regulated by the serotonergic cell circuitry in itself, serotonergic cells being located in various brain areas and interconnected, so that the action of a given serotonergic compound is very difficult to predict and understand. The final outcome of pharmacological modulation within the serotonergic system depends from some internal changes on the interaction between serotonergic neurons [19]. Systematic approaches to define small localised modular networks as part of larger networks to more specifically explain psychopathology [20], seem interesting but are just beginning.

In conclusion, the unspecificity of organic psychosyndromes represents a yet unsolved challenge at the symptom level, which appears to a considerable extent peculiar to the brain organisation itself. A diagnosis of organic psychosyndromes can be made by combining clinical and technical approaches to one patient and evaluating all phenomena and findings in one. This means for the clinical approach to the patient that diagnosing an organic psychosyndrome will continue to need both, extraordinary clinical experience and technical expertise, usually by bringing experts together to evaluating one patient's disease to come to valid conclusions. The categorization proposed by G. Huber is especially important for second-rank organic psychosyndromes. An underrecognition of second-rank organic psychosyndromes in the clinical approach may have negative consequences for the patient.

\section{PSYCHOLOGICAL AND ORGANIC INTER- ACTION}

Another important, rather unsolved aspect of causality inference and categorization of 'organic' aspects of psychosyndromes is, that the psychological level of functioning is apparently in itself able to establish 'organic' consequences or phenomena: this was recently shown in many studies with functional magnetic resonance imaging (fMRI), but nevertheless long known: the will or the psychological activity can lead to 'organic' consequences, first shown with the Bereitschaftspotential [21, 22]. Nevertheless it is interesting to see that some 'low level organic changes' within the brain as demonstrated in a body of evidence for posttraumatic stress disorder, was shown to be possibly reversed by psychotherapy in somatoform disorder [23]. A recent surprising demonstration of long-term consequences of psychological insults was that stressful family environment may lead to enduring changes in neurodevelopment, cortical thickness was increased yet in adulthood [24]. Possible psychological consequences of variant neurodevelopment have also been shown [18]. Should we then call such changes 'organic'? Probably or somehow yes. Nevertheless, there are likely limitations for such psychological to organic interaction. Of note is also that organic pathologies of the brain may not necessarily have any psychopathological consequences.

\section{CLINICAL DIAGNOSIS OF NEUROINFLAM- MATION}

Organic causes of psychosyndromes include the category of neuroinflammatory disorders. Acute neuroinflammation is described as encephalitis or meningoencephalitis which may after four weeks be diagnosed as chronic. A generally accepted time-frame is however not established and may not be justified regarding the biological mechanisms, which generally differ between acute and chronic neuroinflammation [25] but not necessarily by defined timeframe.

Classical acute encephalitis or meningoencephalitis is characterized by typical symptoms including neurological symptoms, which in initial stages may be minor or just beginning. The clinical examination is completed by technical findings: imaging is most convenient and nearly always applied, allowing in part of the cases yet a final diagnosis though nor specific etiologies can be assessed nor imaging represent the most sensitive approach [26]. The gold standard, i.e. the most sensitive approach to diagnose meningoencephalitis, remains CSF investigation. Differential diagnosis of encephalitis is rather difficult, many agents and causes including toxic causes are to be considered. Chronic neuroinflammation and low level neuroinflammation is more difficult to assess. To interpret CSF findings, though characteristic in acute meningoencephalitis, less easy in acute encephalitis without meningitis and very difficult in low level neuroinflammation, needs specific education. With expertise but which cannot be outlined here relatively safe conclusions can be made, (compare [27]). Some important principle might be described, especially that from recent developments of CSF analysis in clinical neurology, which were adopted to clinical psychiatry [28]: investigate always paired CSFserum samples taken at one time point from the patient, allowing to evaluate the dynamics of exchange between the blood and CSF compartment at specific time point. In both compartments all immune globulin subclasses should be investigated and the respective quotients compared to Qalbumin. These CSF/serum quotients with new hyperbolic functions overcome the previous linear functions or formula or indexes, the latter not in all conditions exact. A final classification of each case then will be made by involving all parameters evaluated in one set of CSF/serum findings including cells, an approach helpful to generally reduce false positive findings. Outliers and errors within one dataset or implausible laboratory findings are more likely to be identified when seeing all data in one case at once. By such approach, inflammatory versus non-inflammatory CSFs can be differentiated rather well. A non-inflammatory CSF nevertheless cannot absolutely exclude cases of even an acute encephalitis. Such has been learned from illustrating clinical 
cases in longstanding experience in clinical neurology. For the cerebral cortex or the CSF from subarachnoid spaces above the tentorium, the lumbar CSF investigation may not be sensitive (compare [27, 29]). This likely relates to the general CSF flow patterns and CSF recirculation pathways [30].

In acute encephalitis, the various specific etiologies can be detected rather sensitively by PCR methods in the first week of disease, or less sensitive in later stages, by assessing specific antibody CSF/serum indices (compare [31]). However, a negative PCR finding or a normal specific antibody index does not exclude a specific etiology [27]. Just to mark where we stand. Even in large recent studies attempting to improve the differential diagnosis of acute encephalitis, a bit less than $50 \%$ of cases were clearly classified by etiology [32]. In meningoencephalitis apparently, yet unknown infectious agents seem to prevail.

In chronic (meningo-)encephalitis the sensitivity of methods including CSF investigation is considerably less clear and less conclusive. So such a diagnosis may be more arbitrary also for psychosyndromes. For the most relevant bacteria leading to chronic meningoencephalitis, borrelia species, treponema pallidum, mycobacteria, tuberculosis and listeria monocytogenes, there are relatively well established signs and findings. Nevertheless, a major debate is going on about the sensitivity of the methods in these cases, even when clear neurological symptoms were present or accompanying symptoms like severe chronic pain and other symptoms of chronic meningoencephalitis prevailed. The technical programs, i.e. the approach to the single patient for a safe diagnosis are fluent, but the more a sophisticated knowledge basis of CSF evaluation is important. For example, a pure blood-CSF-barrier dysfunction may suggest low level neuroinflammation when other explanations in a clinical framework view appear unlikely (compare [33]). Or the type of the intrathecal immune class response may be characteristic for certain diseases like tuberculosis or neuroborreliosis. So by combining various parameters and including case history and findings, a more and more specific conclusion in the single case can be drawn. For neuroborreliosis a characteristic data pattern is a dominant IgM class intrathecal immune response combined with blood-CSF-barrier dysfunction increased cell count and normal lactate (compare [34]). Other unspecific chemokine or cytokine findings were just reported as characteristics [35]. Other unspecific parameters are useful in other diseases (compare [27]).

Such laboratory aspects can however the least well be approved in research on organic psychosyndromes associated with neuroinflammation, because many cases may show only low level neuroinflammation and because of the general unspecificity of symptoms described above. Nevertheless, in severe neuroinflammation often first-rank organic symptoms may present as part of the syndrome some time. However, pure psychopathological syndromes without neurological symptoms or other types of second-rank organic psychosyndromes (see above), is much more difficult to diagnose as organic or inflammatory. Thus, it is important to adopt the most advanced methods known in neurological syndromes, and to include interdisciplinary knowledge about the approach to neuroinflammation into conclusions regarding inflammatory or infectious organic psychosyndromes. When combining clinical findings and technical findings and evaluating even borderline aberrations of these measures with respect to time course of psychopathology, one may come to plausibility conclusions, then important for therapeutic approaches.

\section{SPECIAL CASE: THE MILD ENCEPHALITIS (ME) HYPOTHESIS}

A major part of severe psychiatric disorders, the previously so-called endogenous or idiopathic psychosis may and seems to be related to some underlying organic basis according to the opinion of many psychiatric researchers. Nevertheless, it remained very difficult to pinpoint such underlying causes. Immune abnormalities and insults from various infectious agents have been proposed to be involved, eventually low level neuroinflammation seems to explain a considerable subgroup of cases with severe psychiatric disorders from the affective and the schizophrenic type [10]. Therapeutic approaches in therapy-resistant cases with depression or schizophrenia are beginning, although no diagnostic parameters were approved up to now. In own studies we recently found in more than $40 \%$ of cases with affective or schizophrenic disorders certain CSF abnormalities best interpreted as indicating low level neuroinflammation, thus supporting the ME hypothesis in a considerable subgroup of cases [33]. This was complemented by finding abnormal activation patterns of CSF cells, despite normal CSF cell numbers, in part of these cases [36]. Abnormal CSF rather strongly indicates some abnormality within the brain. One can rather firmly assume that in patients with low level inflammatory CSF and prevailing psychosis, the patient may suffer from a low level neuroinflammatory disease i.e., neuroinflammation to represent the cause or pathogenetic basis of the respective psychiatric disorder. The unspecificity rule outlined above, should especially in low level neuroinflammation be taken in mind. The ME hypothesis was further supported by recent results from experimental therapeutic studies, with COX2 blockers [37], CSF filtration [38, 39], and neuroprotective [40-42], as well as immune-suppressive approaches [43].

\section{CSF AND NEUROINFLAMMATION}

But what is the role of CSF in neuroinflammation? According to recent neuroimmunological insights, the CSF spaces probably represent a previously underestimated interface between periphery and CNS, playing an important role in immune surveillance in health and disease [44]. This recent interpretation is backed by the longstanding clinical experience in neurology where apparently the CSF spaces are of extraordinary importance for diagnosis of neuroinflammation. According to recent studies in the initial phase of neuroinflammation, for example in experimental allergic encephalomyelitis, inflammation begins at the meninges, i.e. close to or within the subarachnoid spaces and CSF [45]. What happens during neuroinflammation within the brain during various infections is also increasingly studied in detail. The mechanisms are surprisingly complex, differing in 
between various infections in remarkable aspects, and surely these mechanisms not always do fulfil the classical definition of inflammation [46]. Modern definitions of inflammation in general are beyond previous classical inflammation involving a complex set of soluble and cellular factors [47]. Different types of low level neuroinflammation have been mentioned, however, criteria to differentiate low level from classic neuroinflammation are not available, though proposals were made. For example, in experiments the consequences of toxic insults within the CNS are called inflammatory but clearly not covered by classical definitions of neuroinflammation [48]. The basis for the clinical term 'mild encephalitis' and the ME hypothesis (compare [49]) was a multitude of evidences from clinical and experimental findings that low level neuroinflammation may prevail though not easily be diagnosed by available methods in the clinic, but nevertheless may from a body of experimental evidence be able to induce a variety of psychiatric and neuropsychiatric symptoms, especially a subgroup of affective and schizophrenic disorders [49]. Such view appears increasingly supported now [10].

\section{OUTLOOK}

The concepts in immunology, also with respect to neuroimmunology are in rapid progress. For example, the role of the blood-brain-barrier [50], and the function of the bloodCSF-barrier [51, 52], are newly understood. This bears important consequences for the understanding of neuroinflammation in general. The role of meningeal vessels in early neuroinflammation was only recently understood and with this the role of the CSF spaces. A poorly investigated aspect is however that of CSF signalling and recirculation. CSF signalling and flow characteristics could play an important role in the pathophysiology of neuropsychiatric diseases including neurodegeneration [53]. CSF signalling however may not only be involved in the pathogenesis of symptoms by involving all CNS structures lying within the subarachnoid spaces, or close to the subarachnoid spaces, but may even induce peripheral symptoms via the peripheral cerebrospinal fluid outflow pathways (PCOP), for example possibly involved in polyneuropathy or dysautonomia or muscle lesions found in psychiatric disorders [30]. A number of poorly explained clinical symptoms such as diffuse pain in depression or in fibromyalgia, or similar but more severe symptoms in classical acute meningoencephalitis may be explained by such an interaction between pathological CSF contents and nerve roots, and in addition with brain nerves and peripheral nerves along the PCOP. The physiological cell trafficking into the CSF spaces [54-56] is apparently important in health and disease [44].In contrast, CSF trafficking out of the CSF spaces is not well studied yet. This may be a considerable gap of knowledge also for pathophysiology. Recently we were able to demonstrate for the first time in a rare single case that leukaemia cells apparently followed the PCOP and induced local infiltrations of nerves and skin [57]. In conclusion, neuroinflammation and especially low level neuroinflammation bears many unsolved questions. CSF investigation seems a key to better understand neuroinflammation, especially in the clinical approach.

\section{CONFLICT OF INTEREST}

The authors confirm that this article content has no conflicts of interest.

\section{ACKNOWLEDGEMENTS}

Declared none.

\section{REFERENCES}

[1] Bechter K. Personality disorders improved after arachnoid cyst neurosurgery, then rediagnosed as 'minor' organic personality disorders. Psychiatry Res 2010; 184(3): 196-200.

[2] Huber G. Ed. Psychiatrie. Lehrbuch für Studierende und Ärzte. $5^{\text {th }}$ ed. Stuttgart: Schattauer GmbH 1998.

[3] Gross G, Huber G, Lin M. Zur Frage der symptomatischen Schizophrenie und Zyklothymie. Zbl Neurol 1989; 251: 323-32.

[4] Casey P, Brendan K, Eds. Fish's clinical psychopathology. signs and symptoms in psychiatry. Glasgow, UK: Gaskell 2007.

[5] Buchsbaum MS, Rieder RO. Biologic heterogeneity and psychiatrict research. Platelet MAO activity as a case study. Arch Gen Psychiatry 1979; 36(11): 1163-9.

[6] Kaplan HI, Sadock BJ, Eds. Kaplan and Sadock's Synopsis of Psychiatry Behavioral Sciences/Clinical Psychiatry. $8^{\text {th }}$ ed. Williams \& Wilkins: Baltimore, Md 1998.

[7] van Praag HM. Nosologomania: a disorder of psychiatry. World J Biol Psychiatry 2000; 1(3): 151-8.

[8] Bechter K. Editorial: Interactions of biological and psychological factors in psychopathology - A remaining challenge. Neurol Psychiatry Brain Res 2011; 17(2): 37-38.

[9] Wexler BE. Beyond the Kraepelinean dichotomy. Biol Psychiatry 1992; 31(6): 539-41.

[10] Bechter K, Updating the mild encephalitis hypothesis of schizophreania. Prog neuropsychopharmacol. Biol Psychiatry. In press doi: 10.1016/j.pnpbp.2012.1006.1019.

[11] Giedd JN. Neuroimaging of pediatric neuropsychiatric disorders: Is a picture really worth a thousand words? Arch Gen Psychiatry 2001; 58(5): 443-4.

[12] Newport DJ, Nemeroff CB. Depression in the medically ill. In: Tohen M, Ed. Comorbidity in Affective Disorders. New York: Marcel Dekker 1999; pp. 57-104.

[13] Bonhoeffer K. Die exogenen Reaktionstypen. Arch Psychiat Nervenkr 1917; 58: 58-70.

[14] Niedermeyer E, Ribeiro M, Hertz S. Mixed-type encephalopathies: preliminary considerations. Clin Electroencephalogr 1999; 30(1): 12-5.

[15] Davidson RJ, Putnam KM, Larson CL. Dysfunction in the neural circuitry of emotion regulation--a possible prelude to violence. Science 2000; 289(5479): 591-4.

[16] Herpertz SC. et al. Emotion in criminal offenders with psychopathy and borderline personality disorder. Arch Gen Psychiatry 2001; 58(8): 737-45.

[17] Meyer U. et al. The time of prenatal immune challenge determines the specificity of inflammation-mediated brain and behavioral pathology. J Neurosci 2006; 26(18): 4752-62.

[18] Coid JW. Aetiological risk factors for personality disorders. Br J Psychiatry 1999; 174: 530-8.

[19] Tremblay P, Blier P. Catecholaminergic strategies for the treatment of major depression. Curr Drug Targets 2006; 7(2): 149-58.

[20] Seitz RJ, Gaebel W, Zielasek J. Modular networks involving the medial frontal cortex: towards the development of neuropsychiatry. World J Biol Psychiatry 2011; 12(4): 249-59.

[21] Kornhuber HH, Deecke L. Hirnpotentialänderungen beim Menschen vor und nach Willkürbewegungen und passiven Bewegungen des Menschen, dargestellt mit Magnetbandspeicherung und Rückwärtsanalyse. Pflügers Arch Physiol 1964; 281: 52.

[22] Kornhuber HH, Deecke L, Eds. Wille und Gehirn. 2007 ed. Bielefeld und Locarno: Sirius 2007. 
[23] de Greck M. Multimodal psychodynamic psychotherapy induces normalization of reward related activity in somatoform disorder. World J Biol Psychiatry 2011; 12(4): 296-308.

[24] Benedetti F. et al. Emotional reactivity in chronic schizophrenia: structural and functional brain correlates and the influence of adverse childhood experiences. Psychol Med 2011; 41(3): 509-19.

[25] Amor S. Inflammation in neurodegenerative diseases. Immunology 2010; 129(2): 154-69.

[26] Osborn AG, Salzman KL, Blaser SI, Eds. Diagnostic Imaging Brain. Oxford: Elsevier LTD 2004.

[27] Wildemann B, Oschmann P, Reiber H, Eds. Laboratory Diagnosis in Neurology. Stuttgart: Thieme 2010.

[28] Reiber H, Otto M, Bechter K. Crash Course: Cerebrospinal Fluid Diagnostics for Psychiatrists and Neurologists. Cerebrospinal fluid analysis for diagnosis of non-inflammatory, dementive and psychiatric diseases. Acta Neuropsychiatr 2009; 21 (Suppl 2): 5861.

[29] Felgenhauer K, Beuche W, Eds. Labordiagnostik neurologischer Erkrankungen. Liquoranalytik und Zytologie, Diagnose- und Prozessmarker. Stuttgart: Thieme 1999.

[30] Bechter K. The peripheral cerebrospinal fluid outflow pathway physiology and pathophysiology of CSF recirculation: a review and hypothesis. Neurol Psychiatry Brain Res 2011; 17(3): 51-66.

[31] Reiber H, Peter JB. Cerebrospinal fluid analysis: disease-related data patterns and evaluation programs. J Neurol Sci 2001; 184(2): 101-22.

[32] Glaser CA. et al. Beyond viruses: clinical profiles and etiologies associated with encephalitis. Clin Infect Dis 2006; 43(12): 1565-77.

[33] Bechter K. et al. Cerebrospinal fluid analysis in affective and schizophrenic spectrum disorders: identification of subgroups with immune responses and blood-CSF barrier dysfunction. J Psychiatr Res 2010; 44(5): 321-30.

[34] Tumani H, Nolker G, Reiber H. Relevance of cerebrospinal fluid variables for early diagnosis of neuroborreliosis. Neurology 1995; 45(9): 1663-70.

[35] Rupprecht TA. et al. The chemokine CXCL13 is a key regulator of $B$ cell recruitment to the cerebrospinal fluid in acute Lyme neuroborreliosis. J Neuroinflammation 2009; 6: 42.

[36] Maxeiner HG. et al. Flow cytometric analysis of T cell subsets in paired samples of cerebrospinal fluid and peripheral blood from patients with neurological and psychiatric disorders. Brain Behav Immun 2009; 23(1): 134-42.

[37] Muller N. et al. The cyclooxygenase-2 inhibitor celecoxib has thera-peutic effects in major depression: results of a double-blind, randomized, placebo controlled, add-on pilot study to reboxetine. Mol Psychiatry 2006; 11(7): 680-4.

[38] Bechter K. et al. Borna disease virus-related therapy-resistant depression improved after cerebrospinal fluid filtration. J Psychiatr Res 2000; 34(6): 393-6.

[39] Bechter K. et al. CSF Filtration as Experimental Therapy in Therapyresistant Psychoses in Borna Disease Virus-Seropositive Patients. Psychiatr Prax 2003; 30(Suppl 2): 216-20.
[40] Ehrenreich H. et al. Recombinant human erythropoietin in the treatment of human brain disease: focus on cognition. J Ren Nutr 2008; 18(1): 146-53.

[41] Ehrenreich, H. et al. Exploring recombinant human erythropoietin in chronic progressive multiple sclerosis. Brain 2007; 130(Pt 10): 2577-88.

[42] Ehrenreich $\mathrm{H}$. et al. Improvement of cognitive functions in chronic schizophrenic patients by recombinant human erythropoietin. Mol Psychiatry 2007; 12(2): 206-20.

[43] Knight JG. Rationale for a trial of immunosuppressive therapy in acute schizophrenia. Mol Psychiatry 2007; 12(5): 424-31.

[44] Schwartz M, Shechter R. Protective autoimmunity functions by intracranial immunosurveillance to support the mind: The missing link between health and disease. Mol Psychiatry 2010; 15(4): 34254.

[45] Brown DA, Sawchenko PE. Time course and distribution of inflammatory and neurodegenerative events suggest structural bases for the pathogenesis of experimental autoimmune encephalomyelitis. J. Comp.Neurol 502(2):236-260.

[46] Bentivoglio M, Mariotti R, Bertini G. Neuroinflammation and brain infections: historical context and current perspectives. Brain Res Rev 2011; 66(1-2): 152-73.

[47] Nathan C. Points of control in inflammation. Nature 2002; 420(6917): 846-52.

[48] O'Callaghan JP, Sriram K, Miller DB. Defining "neuroinflammation". Ann N Y Acad Sci 2008; 1139: 318-30.

[49] Bechter K. Mild encephalitis underlying psychiatric disorder - A reconsideration and hypothesis exemplified on Borna Disease. Neurol Psychiatry Brain Res 2001; 9: 55-70.

[50] Bechmann I, Galea I, Perry VH. What is the blood-brain barrier (not)? Trends Immunol 2007; 28(1): 5-11.

[51] Wolburg H, Paulus W. Choroid plexus: biology and pathology. Acta Neuropathol 2010; 119(1): 75-88.

[52] Marques F. et al. The choroid plexus response to a repeated peripheral inflammatory stimulus. BMC Neurosci 2009; 10: 135.

[53] Holman DW, Kurtcuoglu V, Grzybowski DM. Cerebrospinal fluid dynamics in the human cranial subarachnoid space: an overlooked mediator of cerebral disease. II. In vitro arachnoid outflow model. J R Soc Interface 2010; 7(49): 1205-18.

[54] Ransohoff RM. Immunology: In the beginning. Nature 2009; 462(7269): 41-2

[55] Goverman, J. Autoimmune T cell responses in the central nervous system. Nat Rev Immunol 2009; 9(6): 393-407.

[56] Engelhardt B, Sorokin L. The blood-brain and the bloodcerebrospinal fluid barriers: function and dysfunction. Semin Immunopathol 2009; 31(4): 497-511.

[57] Schmitt M. et al. Spreading of acute myeloid leukemia cells by trafficking along the peripheral outflow pathway of cerebrospinal fluid. Anticancer Res 2011; 31(6): 2343-5.

\section{(C) Karl Bechter; Licensee Bentham Open.}

This is an open access article licensed under the terms of the Creative Commons Attribution Non-Commercial License (http://creativecommons.org/licenses/by-nc/3.0/) which permits unrestricted, non-commercial use, distribution and reproduction in any medium, provided the work is properly cited. 